\title{
Morphology and luminosity segregation of galaxies in nearby loose groups
}

\author{
M. Girardi, E. Rigoni, F. Mardirossian, and M. Mezzetti
}

Dipartimento di Astronomia, Università degli Studi di Trieste, Via Tiepolo 11, 34131 Trieste, Italy

e-mail: [girardi,rigoni, mardiros, mezzetti]@ts. astro.it

Received 13 March 2003 / Accepted 19 May 2003

\begin{abstract}
We study morphology and luminosity segregation of galaxies in loose groups. We analyze the two catalogs of groups identified in the Nearby Optical Galaxy (NOG) sample, by means of hierarchical and percolation "friends-of-friends" methods (HG and PG catalogs, respectively). In the first part of our analysis we consider 387 and 436 groups of HG and PG and compare morphology- (luminosity-) weighted to unweighted group properties: velocity dispersion, mean pairwise distance, and mean groupcentric distance of member galaxies. The second part of our analysis is based on two ensemble systems, one for each catalog, built by suitably combining together galaxies of all groups (1584 and 1882 galaxies for HG and PG groups). We find that earlier-type (brighter) galaxies are more clustered and lie closer to the group centers, both in position and in velocity, than later-type (fainter) galaxies. Spatial segregations are stronger than kinematical segregations. These effects are generally detected at the $\gtrsim 3$-sigma level. Luminosity segregation is shown to be independent of morphology segregation. Our main conclusions are strengthened by the detection of segregation in both hierarchical and percolation catalogs. Our results agree with a continuum of segregation properties of galaxies in systems, from low-mass groups to massive clusters.
\end{abstract}

Key words. galaxies: clusters: general - galaxies: fundamental parameters - galaxies: evolution - cosmology: observations

\section{Introduction}

Groups and clusters of galaxies are complex systems involving a variety of interacting components (galaxies, X-ray emitting gas, dark matter). Their investigation offers a rare opportunity to link many aspects of astrophysics and cosmology and, in particular, to clarify the interplay between dark and baryonic matter.

Different galaxy populations, i.e. families of galaxies with different morphology - color - spectral type - luminosity, show different distributions in projected position and LOS velocity. These phenomena, known as segregation effects, provide a way to explore the connection between the distributions of dark matter and galaxies.

Segregation phenomena are well studied in galaxy clusters. Since the first studies (e.g. Oemler 1974; Moss \& Dickens 1977; Dressler 1980; Dressler et al. 1997), a long sequence of analyses has shown that galaxies of early morphological type (red color - low star formation rate) are more concentrated in regions of higher projected density and lie closer to the cluster center both in position and in velocity than galaxies of late morphological type (blue color - high star formation rate), cf. Biviano et al. (2002) and references therein. Evidence for luminosity segregation is also found, although only very luminous,

Send offprint requests to: M. Girardi, e-mail: girardi@ts.astro.it possibly early-type galaxies seem really to be segregated from the rest of the population (e.g. Biviano et al. 1992; Stein 1997; Biviano et al. 2002).

Observational evidence that in galaxy surveys the clustering strength depends on morphology, luminosity, and colors (e.g. Benoist et al. 1996; Hermit et al. 1996; Guzzo et al. 1997; Norberg et al. 2001) suggests that segregation phenomena in galaxy systems might be connected with the large-scalestructure formation, perhaps in the context of biased galaxy formation or in the hierarchical growth of structure via gravitational instability.

Alternatively and/or additionally, some environmental effects could play an important rôle in segregation phenomena (e.g. White 1983; Richstone 1990; Moss 2001). In fact, after a fast stage of violent relaxation, when the dynamics is controlled by a collective potential (Lynden-Bell 1967), galaxy systems should undergo a secondary relaxation phase, characterized by a longer time scale. In this second phase, several physical effects could modify member galaxies as regards their internal properties, as well as their distribution in space and in velocity. Some of these environmental effects, such as ram pressure stripping (Gunn \& Gott 1972) and galaxy harassment (Moore et al. 1996), are less effective in group environments, where the $\mathrm{X}$-ray temperature and global potential are smaller than in clusters. On the contrary, galaxy-galaxy interactions, such as close tidal encounters or mergers, and dynamical friction should be 
particularly significant in poor groups, where the velocity dispersion is lower than in clusters (e.g. Sarazin 1986; Richstone 1990; Makino \& Hut 1997).

In this context, differences in the segregation effects between groups and clusters would suggest that the system environment is fundamental in transforming galaxies at the present epoch. On the contrary, no difference might rather suggest that galaxy properties are influenced by initial conditions at the time of galaxy formation, maybe through the hierarchical growth of structures.

Unfortunately, observational difficulties prevented researchers from giving a precise description of segregation effects in poor galaxy systems.

The only strongly supported effect in groups is the spatial segregation of galaxies of different morphological type (color - spectral type), which is the best known effect in clusters. Pioneering investigations were performed by Ozernoy \& Reinhard (1976), Bhavsar (1981), and de Souza et al. (1982). The first systematic results using groups with redshift information come from the study of Postman \& Geller (1984). Through the analysis of groups in the CfA Redshift Survey and in the Catalog of Nearby Galaxies they showed that the relationship between galaxy morphology and local density, as found for rich clusters, extends down to groups. The presence of morphological segregation in groups of the CfA survey was also confirmed by Mezzetti et al. (1985) and Giuricin et al. (1988), who computed the mean groupcentric distance and the mean pairwise distance of the members in each individual group for different galaxy populations. Further segregation evidence comes from the analysis of the groups identified in the Southern Sky Redshift Survey (Maia \& da Costa 1990).

Recent works support the existence of morphology segregation phenomena, too. Mahdavi et al. (1999) analyzed 20 well sampled groups finding that galaxies of different spectral types are segregated in space. Tran et al. (2001) made a deep analysis of six X-ray detected groups finding that bulgedominated galaxies decreases with increasing radius, similar to the morphology-radius relation observed in clusters. Carlberg et al. (2001b) analyzed $\sim 200$ groups at intermediate redshift $(z=0.1-0.55)$ identified within the CNOC2 (Canadian Network for Observational Cosmology's field galaxy redshift survey) and found the presence of color gradients, i.e. the galaxies are redder toward the group center, in the most massive groups. Domínguez et al. (2002) analyzed the $2 \mathrm{dF}$ Group Catalog and found that the fraction related to low starformation galaxies depends on the local density and the groupcentric radius in the most massive $(\sim 40)$ groups.

Kinematical segregation of galaxies of different morphological types is found in galaxy clusters (e.g. Stein 1997; Biviano et al. 2002), but it is a smaller effect with respect to spatial segregation. In fact, the most different population seems to be the $10 \%$ of galaxies with strong emission lines - ELGs which have considerably larger global velocity dispersion than other galaxy populations (de Theije \& Katgert 1999; Biviano et al. 1997). The question is still open for groups: galaxies of different spectral types seem to differ in the value of of velocity anisotropy, but not in the value of the global velocity dispersion (Mahdavi et al. 1999).
As for luminosity segregation in galaxy clusters, low levels of significance are found both in position and in velocity. In fact, this kind of segregation concerns only very few luminous galaxies or only early-type galaxies (e.g., Biviano et al. 1992, 2002; Stein 1997; Adami et al. 1998). As for groups, the results are generally obtained by comparing luminosity-weighted and unweighted properties of galaxy groups. Ozernoy \& Reinhard (1976) found that the effect of luminosity weighting is to increase the harmonic radius and to lower the velocity dispersion in comparison with the unweighted values. Further analyses of more recent group catalogs do not come on a common conclusion. Giuricin et al. (1982) claimed that the virial parameters are largely insensitive to weighting procedures, suggesting that group galaxies are in a status of velocity equipartition, while Mezzetti et al. (1985) found that virial radii are affected by weighting. Recent evidence for luminosity segregation in space and in velocity comes from the study of Magtesyan \& Movsesyan (1995, 303 groups), but with small significance $(\$ 95 \%)$.

In this framework, we analyze the presence of segregation effects in galaxy groups identified in the Nearby Optical Galaxy Catalog (NOG, Giuricin et al. 2000, hereafter G00), which samples the local Universe. The advantage of using NOG groups is threefold: 1) the amount of morphological information available for nearby galaxies; 2) the presence of many low-mass local groups in this all-sky catalog; 3) the availability of two alternative catalogs of groups identified in the same galaxy catalog using two different algorithms. On the other hand, these groups suffer from the problem plaguing traditional catalogs, i.e. the poor statistics available for each group. In view of this problem, we have devoted some effort to combining the data of many groups.

The paper is organized as follows. We describe the data sample and compute the main physical group quantities in Sect. 2. We devote Sects. 3 and 4 to the detection and analysis of the segregation effects. We discuss our results and draw our conclusions in Sect. 5.

Unless otherwise stated, we give errors at the $68 \%$ confidence level (hereafter c.1.).

A Hubble constant of $100 h \mathrm{~km} \mathrm{~s}^{-1} \mathrm{Mpc}^{-1}$ is used throughout.

\section{Data sample and group properties}

We analyze the galaxy loose-groups identified in the NOG catalog by G00. NOG is a complete apparent-magnitude catalog (corrected total blue magnitude $\mathrm{B} \leq 14$ ), with an upper distance limit $\left(c z<6000 \mathrm{~km} \mathrm{~s}^{-1}\right)$, and collects $\sim 7000$ optical galaxies, basically extracted from the Lyon-Meudon Extragalactic Database (LEDA; c.f. Paturel et al. 1997). NOG covers about $2 / 3$ of the sky $\left(|b|>20^{\circ}\right)$, and is quasi-complete in redshift (97\%). Almost all NOG galaxies (98.7\%) have a morphological classification as taken from LEDA, and parameterized by $T$ (the morphological-type code system of RC3 catalog de Vaucouleurs et al. 1991) with one decimal figure.

G00 identified NOG groups by means of both the hierarchical and the percolation "friends-of-friends" methods. In particular, they employed two variants of the percolation method, 
Table 1. Group properties.

\begin{tabular}{lccccrrrr}
\hline \hline Sample & $N_{\text {GROUPs }}$ & $N_{\text {GALs }}$ & $n$ & $z$ & $\begin{array}{c}\sigma_{\mathrm{v}} \\
\mathrm{km} \mathrm{s}^{-1}\end{array}$ & $\begin{array}{c}R_{\mathrm{V}} \\
h^{-1} \mathrm{Mpc}\end{array}$ & $\begin{array}{c}\mathcal{M} \\
h^{-1} \\
10^{13} \mathcal{M}_{\odot}\end{array}$ & $R_{\max } / R_{\text {vir }}$ \\
\hline HG & 387 & 2017 & 4 & 0.012 & $89_{-6}^{+5}$ & $0.61_{-0.05}^{+0.04}$ & $0.5_{-0.1}^{+0.1}$ & 3.48 \\
HG5 & 148 & 1216 & 6 & 0.010 & $104_{-8}^{+8}$ & $0.65_{-0.06}^{+0.05}$ & $0.7_{-0.1}^{+0.2}$ & 3.98 \\
PG & 436 & 2262 & 4 & 0.012 & $138_{-1}^{+12}$ & $0.47_{-0.03}^{+0.05}$ & $0.9_{-0.1}^{+0.2}$ & 1.61 \\
PG5 & 168 & 1375 & 6 & 0.010 & $162_{-20}^{+17}$ & $0.53_{-0.04}^{+0.04}$ & $1.5_{-0.4}^{+0.3}$ & 1.81 \\
\hline
\end{tabular}

which gave very similar catalogs of groups. Here we use the P2 catalog obtained with the variant where both the distance link parameter and the velocity link parameter scale with distance (Huchra \& Geller 1982), which is the most frequently used method of group identification in the literature. As for the hierarchical method, G00 basically followed the procedure adopted by Gourgoulhon et al. (1992).

The final hierarchical and percolation catalogs contain 475 and 513 groups with at least three members, respectively (hereafter HG and PG). The HG and PG catalogs turn out to be substantially consistent as far as the distribution of members in groups is concerned (see G00 for more details).

The availability of these two alternative samples of groups identified in the same galaxy catalog is of great advantage in our study. In fact, owing to the small number of galaxies for each group, one must rely on members as assigned by the group-selection algorithm, rather than using refined methods to reject interlopers as performed in clusters or very well sampled groups (e.g., 12 groups of Zabludoff \& Mulchaey 1998a; 20 groups of Mahdavi et al. 1999). In particular, the resulting group properties may depend on the choice of the groupselection algorithm and its free parameters (e.g., Pisani et al. 1992; Frederic 1995; Ramella et al. 1997). In fact, in the case of the NOG catalog, although the HG and PG groups have a large overlap in their members, the surviving difference in membership leads to differences in their main properties (cf. Table 1 and Fig. 1). In this context, our conclusions regarding segregation effects, where the main properties are used in an explicit way or in rescaling parameters (cf. Sects. 3 and 4, respectively), will be strengthened by their detection in both hierarchical and percolation catalogs.

We remove from our analysis groups with $c z \leq$ $1000 \mathrm{~km} \mathrm{~s}^{-1}$, because when the velocity becomes low its random component dominates and the velocity is no longer a reliable indication of the distance. In this way we reject most galaxies associated with the Virgo cluster too, i.e. the clumps A and B, the cloud W' and the Southern Extension SE as identified by G00 in accordance with Binggeli et al. (1987) and Binggeli et al. (1993). Moreover, we remove the more distant parts of Virgo ( $\mathrm{M}$ and $\mathrm{W}$ clouds), as well as all groups identified by G00 as known clusters (cf. their Tables 5 and 7). Obviously, we do not consider groups where the correction for observational velocity errors (cf. below) leads to a negative value of the velocity dispersion of member galaxies. Our final samples, HG and PG, contain 387 and 436 groups with at least three members for a total of 2017 and 2262 galaxies, respectively. Of the HG groups, 350 comprise $n<10$ members (in particular 155 have $n=3$ members), 31 groups comprise

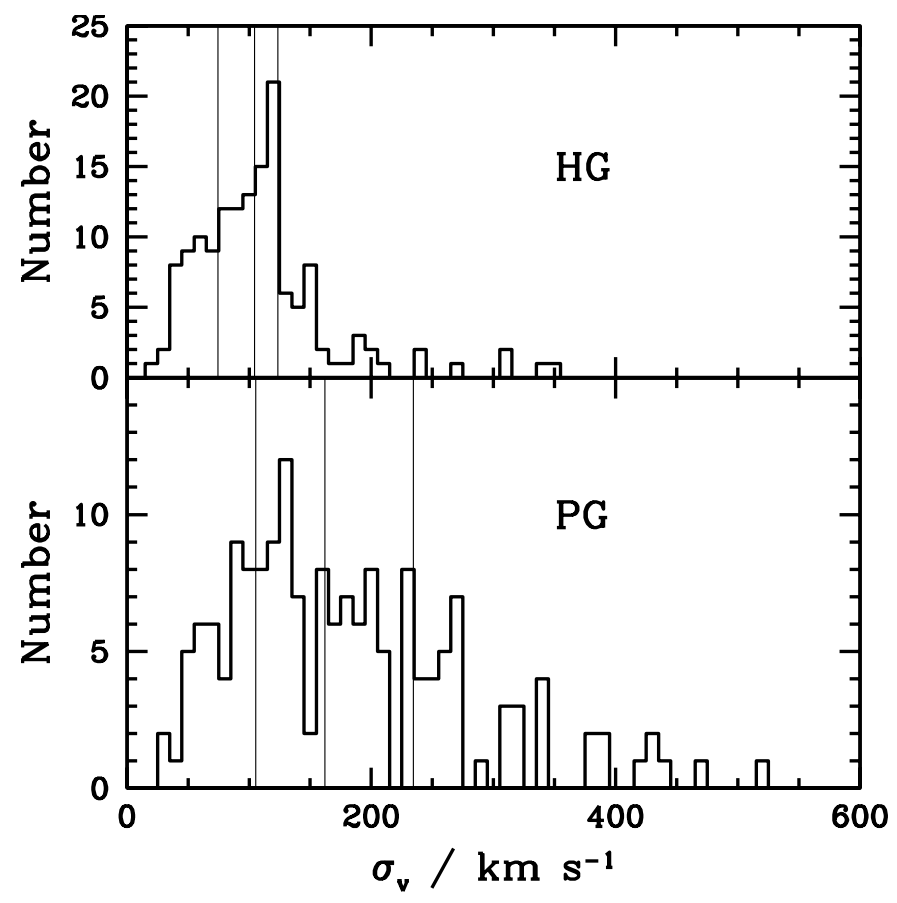

Fig. 1. Histograms of $\sigma_{\mathrm{v}}$-values for rich $(n \geq 5)$ groups in the HG and PG catalogs (top and bottom panels, respectively). The faint vertical lines indicate the separation between the quartiles of the distribution.

$10 \leq n<20$ members, and 6 groups have $n \geq 20$ members. Of the PG groups, 394 comprise $n<10$ members (in particular 185 have $n=3$ members), 36 groups comprise $10 \leq n<20$ members, and 6 groups have $n \geq 20$ members.

Out of the above groups, 148 and 168 groups have $n \geq$ 5 members for a total of 1216 and 1375 galaxies (HG5 and PG5, respectively). The physical reality of very poor groups has often been discussed in the literature. In particular, the efficiency of the percolation algorithm has been repeatedly checked, showing that an appreciable fraction of the poorer groups, those with $n<5$ members, might be false (i.e. represent unbound density fluctuations), whereas the richer groups almost always correspond to real systems (e.g., Ramella et al. 1989; Ramella et al. 1995; Mahdavi et al. 1997; Nolthenius et al. 1997; Diaferio et al. 1999). Throughout the paper we apply our analysis to these statistically more reliable groups, too.

We take from G00 the data available for galaxy positions, redshifts (in the Local Group rest frame), corrected total blue magnitudes, and morphologies. For each group, we compute the main physical quantities.

We calculate the mean group velocity $\bar{v}$ by using the biweight estimator (Beers et al. 1990). We also compute the 
biweight group center by the mean of member positions, i.e. we perform the biweight mean-estimator of member rightascensions and declinations, separately. The group size is then given by $R_{\max }$, which is the (projected) distance of the most distant galaxy from the group center.

The main property of a galaxy system is the LOS velocity dispersion, $\sigma_{\mathrm{v}}$. In fact, the virial radius $R_{\mathrm{vir}}$, which defines the region where the matter overdensity is $\sim 178$ (for a CDM $\Omega_{\mathrm{m}}=1$ cosmology), scales with $\sigma_{\mathrm{v}}$, and the mass in the virialized region $\mathcal{M}\left(<R_{\text {vir }}\right)$ scales with $\sigma_{\mathrm{v}}^{3}$ (e.g., Carlberg et al. 1997; Girardi et al. 1998; Antonuccio-Delogu et al. 2002). We estimate $\sigma_{\mathrm{v}}$ by using the biweight estimator for groups with $n \geq 15$ and the gapper estimator for groups with $n<15$ (Beers et al. 1990). We apply the relativistic correction and the usual correction for velocity errors (Danese et al. 1980). In particular, for each galaxy, we assume a typical velocity error of $30 \mathrm{~km} \mathrm{~s}^{-1}$ based on the average errors estimated in the RC3 catalog from optical and radio spectroscopy (de Vaucouleurs et al. 1991).

As for $R_{\mathrm{vir}}$, we adopt the definition by Girardi et al. (1998), recovered by using the observational King-modified galaxy distribution of nearby clusters:

$R_{\mathrm{vir}}=\left[2 \cdot \sigma_{\mathrm{v}} /\left(1000 \mathrm{~km} \mathrm{~s}^{-1}\right)\right] h^{-1} \mathrm{Mpc}$.

The use of an isothermal distribution leads to a similar value (cf. our $R_{\text {vir }}$ with the $R_{200}$ definition by Carlberg et al. 1997).

Another important group quantity is the projected radius $R_{\mathrm{V}}$, which is twice the harmonic radius and is often used in the computation of the virial mass (e.g., Binney \& Tremaine 1987; Girardi et al. 1998):

$\mathcal{M}\left(<R_{\max }\right)=\frac{3 \pi}{2} \cdot \frac{R_{\mathrm{V}} \sigma_{\mathrm{v}}^{2}}{G}$,

where the factor $3 \pi / 2$ is the deprojection factor adequate for spherical systems, and the mass is that contained within the sampled region, i.e. for $R \leq R_{\max }$. This approach to mass estimate is similar to that computed in other group catalogs (e.g., Ramella et al. 2002, and refs. therein), see also Girardi \& Giuricin (2000) for related discussions.

The radius $R_{\mathrm{V}}$ is defined as:

$R_{\mathrm{V}}=n(n-1) / \sum_{i>j} R_{i j}^{-1}$,

where $R_{i j}$ are the projected mutual galaxy distances, and $n$ is the number of group members within the sampled region. The value of $R_{\mathrm{V}}$ depends on the relative galaxy distribution, and the extension of the sampled region, i.e. the region occupied by the galaxies used in the computation.

Table 1 lists the median values (and 90\% confidence intervals) of the above group properties for the HG and PG catalogs. The confidence intervals are computed following the procedure $^{1}$ described by Kendall \& Stuart (1979, eq. 32.23) and first proposed by Thompson (1936). Values for groups with $n \geq 5$ are shown, too.

\footnotetext{
${ }^{1}$ For the median of an ordered distribution of $N$ values the confidence intervals $x_{(r)}$ and $x_{(N-r+1)}$, corresponding to a probability $P\left(x_{(r)} \leq x \leq x_{(N-r+1)}\right)=1-\alpha$, can be obtained from $1-\alpha=$ $2^{-N} \sum_{i=r}^{N-r}\left(\begin{array}{c}N \\ i\end{array}\right)$.
}

Median values of $R_{\mathrm{V}}$ are comparable to typical values quoted in the literature (cf. Table 4 of Tucker et al. 2000, - note that their deprojected $R_{\mathrm{h}}$ corresponds to $\pi / 4 \times R_{\mathrm{V}}$ ).

As for $\sigma_{\mathrm{v}}$, the HG value is $\sim 2 / 3$ of the PG value (cf. also Pisani et al. 1992 for a similar result). Indeed, it has been suggested that the drawback of percolation methods is the inclusion in the catalogs of possible non-physical systems, like a long galaxy filament aligned close to the line of sight, which give large velocity-dispersion estimates, while the drawback of hierarchical methods is the splitting of galaxy clusters into various subunits, which give small velocity-dispersion estimates (e.g., Gourgoulhon et al. 1992). The resulting difference in the distribution of velocity-dispersion estimates is outlined in Fig. 1. Our value of PG $\sigma_{\mathrm{v}}$ lies within the range of values reported by Tucker et al. (2000), which are computed for percolation catalogs, and that of $\mathrm{HG} \sigma_{\mathrm{v}}$ is comparable with results for the hierarchical catalog of Tully (1987).

Note that NOG groups are sampled well outside $R_{\text {vir }}$, in agreement with the conclusion of Girardi \& Giuricin (2000) about groups by Garcia (1993). This characteristic is common in traditional catalogs of loose groups and in fact, Carlberg et al. (2001a) introduced a variant of the friends-of-friends algorithm in such a way as to consider only central, possibly virialized regions of CNOC groups. In Sect. 4 we will consider only central group regions, too.

\section{Detection of segregation}

To detect possible segregation effects in velocity we compare several estimates of velocity dispersions (cf. also Biviano et al. 1992). The unweighted velocity dispersion is defined as:

$\sigma_{\mathrm{u}}=\left[\frac{\sum_{i=1}^{n}\left(v_{i}-\bar{v}\right)^{2}}{n-1}-\Delta\right]^{1 / 2}$,

where $v_{i}$ is the velocity of the $i$ th galaxy corrected for cosmological effects, $\bar{v}=\sum_{i=1}^{n} v_{i} / n$, and $\Delta$ is the correction term which accounts for the measurement errors (see, e.g., Danese et al. 1980).

To check for possible luminosity segregation, we use the luminosity-weighted velocity dispersion:

$\sigma_{\mathrm{lw}}=\left[\frac{\sum_{i=1}^{n}\left(v_{i}-\bar{v}\right)^{2} l_{i}}{\sum_{i=1}^{n} l_{i}} \frac{n}{n-1}-\Delta\right]^{1 / 2}$,

where $l_{i}$ is the apparent galaxy blue luminosity; the groupcentric-distance-weighted velocity dispersion:

$\sigma_{\mathrm{dw}}=\left[\frac{\sum_{i=1}^{n}\left(v_{i}-\bar{v}\right)^{2} R_{i}^{-1}}{\sum_{i=1}^{n} R_{i}^{-1}} \frac{n}{n-1}-\Delta\right]^{1 / 2}$,

where $R_{i}$ is the projected galaxy distance from its groupcenter; and the luminosity-groupcentric-distance-weighted velocity dispersion:

$\sigma_{\mathrm{ldw}}=\left[\frac{\sum_{i=1}^{n}\left(v_{i}-\bar{v}\right)^{2} l_{i} R_{i}^{-1}}{\sum_{i=1}^{n} l_{i} R_{i}^{-1}} \frac{n}{n-1}-\Delta\right]^{1 / 2}$,

where the effects of luminosity- and groupcentric-distanceweighting are coupled. 
Table 2. Comparison of weighted and unweighted group properties.

\begin{tabular}{lccrrrrrr}
\hline \hline & \multicolumn{2}{c}{ HG: 387 systems } & \multicolumn{2}{c}{ HG5: 148 systems } & \multicolumn{2}{c}{ PG: 436 system } & \multicolumn{2}{c}{ PG5: 168 systems } \\
\multicolumn{1}{c}{$X-Y$} & \multicolumn{1}{c}{$P_{\mathrm{S}}$} & \multicolumn{1}{c}{$P_{\mathrm{W}}$} & \multicolumn{1}{c}{$P_{\mathrm{S}}$} & \multicolumn{1}{c}{$P_{\mathrm{W}}$} & \multicolumn{1}{c}{$P_{\mathrm{S}}$} & \multicolumn{1}{c}{$P_{\mathrm{W}}$} & \multicolumn{1}{c}{$P_{\mathrm{S}}$} & \multicolumn{1}{c}{$P_{\mathrm{W}}$} \\
\hline$\sigma_{\mathrm{lw}}-\sigma_{\mathrm{u}}$ & $>99.9$ & $>99.9$ & $>99.9$ & $>99.9$ & $>99.9$ & $>99.9$ & $>99.9$ & $>99.9$ \\
$\sigma_{\mathrm{dw}}-\sigma_{\mathrm{u}}$ & $>99.9$ & $>99.9$ & 98.2 & 98.8 & 99.9 & $>99.9$ & 98.2 & $>99.9$ \\
$\sigma_{\mathrm{ldw}}-\sigma_{\mathrm{u}}$ & $>99.9$ & $>99.9$ & $>99.9$ & $>99.9$ & $>99.9$ & $>99.9$ & 99.6 & $>99.9$ \\
$\sigma_{T_{\mathrm{w}}}-\sigma_{\mathrm{u}}$ & 83.8 & 95.0 & 97.4 & 99.0 & 92.8 & 96.7 & 97.1 & 96.0 \\
$\bar{R}_{\mathrm{lw}}-\bar{R}$ & $>99.9$ & $>99.9$ & $>99.9$ & $>99.9$ & $>99.9$ & $>99.9$ & $>99.9$ & $>99.9$ \\
$\bar{R}_{T \mathrm{w}}-\bar{R}$ & $>99.9$ & $>99,9$ & $>99.9$ & $>99.9$ & $>99.9$ & $>99.9$ & $>99.9$ & $>99.9$ \\
$\bar{R}_{i j \mathrm{lw}}-\bar{R}_{i j}$ & $>99.9$ & $>99.9$ & $>99.9$ & $>99.9$ & $>99.9$ & $>99.9$ & $>99.9$ & $>99.9$ \\
$\bar{R}_{i j, T \mathrm{w}}-\bar{R}_{i j}$ & $>99.9$ & $>99.9$ & $>99.9$ & $>99.9$ & $>99.9$ & $>99.9$ & $>99.9$ & $>99.9$ \\
\hline
\end{tabular}

Moreover, we introduce the morphology-weighted velocity dispersion, $\sigma_{T w}$ :

$\sigma_{T \mathrm{w}}=\left[\frac{\sum_{i=1}^{n}\left(v_{i}-\bar{v}\right)^{2} k_{i}}{\sum_{i=1}^{n} k_{i}} \frac{n}{n-1}-\Delta\right]^{1 / 2}$,

where $k_{i}$ is a weight related to the galaxy morphology: $k=4$ for early-type galaxies and $k=1$ for late-type galaxies, i.e. galaxies with $T<0$ and with $0 \leq T<10$. The choice of a factor of four of difference in the weights is suggested by the typical difference in $M / L_{B}$ ratios for early- and late-type galaxies (e.g. Bahcall et al. 1995).

To detect possible spatial segregation effects, we consider the (projected) mean distance of members from the center of the group:

$\bar{R}=\sum_{i=1}^{n} R_{i} / n$,

and the (projected) mean pairwise separation of the members:

$\bar{R}_{i j}=\frac{\sum_{i>j} R_{i j}}{n(n-1) / 2} ;$

and the respective weighted quantities:

$\bar{R}_{\mathrm{lw}}=\sum_{i=1}^{n} l_{i} R_{i} / \sum_{i=1}^{n} l_{i}$,

$\bar{R}_{T \mathrm{w}}=\sum_{i=1}^{n} k_{i} R_{i} / \sum_{i=1}^{n} k_{i}$,

$\bar{R}_{i j, \mathrm{lw}}=\frac{\sum_{i>j} l_{i} l_{j} R_{i j}}{\sum_{i>j} l_{i} l_{j}}$,

$\bar{R}_{i j, T \mathrm{w}}=\frac{\sum_{i>j} k_{i} k_{j} R_{i j}}{\sum_{i>j} k_{i} k_{j}}$.

We use the Sign and Wilcoxon Signed-ranks tests (hereafter referred to as S- and W-tests, e.g. Siegel 1956) to compare weighed and unweighted estimates of each quantity we compute below. S- and W-tests are nonparametric tests which investigate the median difference between pairs of scores from two matched sample of a certain size. The W-test differs from the S-test in that the magnitude of score differences within pairs is taken into account, rather than simply the direction of such differences.
We find that each weighted quantity is smaller than the respective unweighted quantity. The $\mathrm{S}$ - and $\mathrm{W}$-tests recover a strong significant difference with the exception of $\sigma_{T \mathrm{w}}$, for which only a partial significance is generally found (cf. Table 2). These results agree with the scenario where earliertype (brighter) galaxies are more clustered and lie closer to the group center both in position and in velocity than later-type (fainter) galaxies. Moreover, galaxies which are close to the group center move slowly.

These results are confirmed when only statistically more reliable groups are considered ( $n \geq 5$ members, HG5 and PG5 samples).

Table 2 lists each comparison between weighted and unweighted quantities and gives the percent significance of the difference according to the $\mathrm{S}$ - and $\mathrm{W}$-test $\left(P_{\mathrm{S}}\right.$ and $P_{\mathrm{W}}$, respectively).

From a quantitative point of view: the typical amount of the difference between weighted and unweighted quantities is small, at most $7 \%$ (in the case of $\sigma_{\text {ldw }}$ vs. $\sigma_{\mathrm{u}}$ ). Thus, any segregation effect has a little relevance in the computation of global group quantities. On the contrary, we stress that, as regards the connection between galaxy evolution and environment, the rôle of the above segregation effects is very important and could help to clarify many points. This explains the attempt of the next section and previous analyses to stack together many groups.

\section{Analysis of segregation}

\subsection{Ensemble groups}

Here we analyze the behavior of relations between interesting galaxy properties. Unfortunately, owing to the small number of group members, we cannot address this question by analyzing each individual group. Therefore, we build two ensemble systems, one for each examined catalog, HG and PG, by combining together galaxies of all groups. The procedure of stacking groups of various sizes and masses into an ensemble system requires that individual galaxy quantities are properly scaled.

The magnitudes are normalized to the magnitude of the third-ranked galaxy, $m_{3}$ in each group by using $m-m_{3}$ (cf. also Biviano et al. 1992). Here the median value of $m_{3}$ corresponds to a blue corrected absolute magnitude of $M_{B} \sim-19.5+5 \log h$. We use this normalization to take into account possible biases introduced by the intrinsic nature of the NOG group catalog. 
In fact, members of more distant groups identified in an apparent magnitude galaxy survey are, on average, more intrinsically luminous.

The rest frame LOS velocities are normalized to the global value of velocity dispersion of each group. For each galaxy we consider the absolute quantity $|v-\bar{v}| / \sigma_{\mathrm{v}}$. Note that the standard estimate $\sigma_{\mathrm{u}}$ strongly correlates with the robust estimate $\sigma_{\mathrm{v}}$ (at the $>99.9 \%$ c.l.); thus the results are not affected by the particular choice of velocity-dispersion estimate.

In studies of galaxy clusters, projected groupcentricdistances $R$ are generally rescaled with $R_{\text {vir }}$, whose estimate is proportional to $\sigma_{\mathrm{v}}$ (cf. Sect. 2). However, the question is less obvious for galaxy groups.

Mahdavi et al. (1999) pointed out that, in the case of poor systems, the possible source of errors on the observationally estimated $R_{\text {vir }}$ could be much larger than in the case of clusters. In fact, uncertainties typical of groups, such as their dynamical status and galaxy distribution, add to large uncertainties in the estimate of velocity dispersions, connected to the small number of group members.

Moreover, owing to their different nature, distance and velocity variables are treated in different ways in the group identification algorithms. Thus, if the use of $\sigma_{\mathrm{v}}$ to rescale velocities is fully self-consistent, the use of a quantity proportional to $\sigma_{\mathrm{v}}$ might be not the best choice for rescaling distances.

To further investigate the question, we consider three possible alternative factors for rescaling $R$, i.e. using: 1) $R_{\text {vir }}$, 2) $R_{\mathrm{V}}$, and 3) no rescaling at all. We start from the theoretical prejudice that halo scale-invariance implies that the existence of very different kinds of galaxy distributions for low- and high- $\sigma_{\mathrm{v}}$ groups is an indication of a non-corrected scaling factor. Thus we consider the ensemble systems constructed from low- and high- $\sigma_{\mathrm{v}}$ groups (according to the median value of $\sigma_{\mathrm{v}}$ in Table 1) and we compare the two cumulative distributions of normalized groupcentric-distances through the KolmogorovSmirnov test (K-S test, e.g. Lederman 1982): they are always different, but the smallest amount of difference is found when using $R_{\mathrm{V}}$ (according to the statistic $\mathrm{D}$ of the $\mathrm{K}-\mathrm{S}$ test).

Thus, we choose to rescale groupcentric-distances with $R_{\mathrm{V}}$. However, we have verified that our main results are still valid in the case of the other two choices of normalization.

Figure 2 shows the behavior of magnitude, and morphological-type vs. projected groupcentric distance. To be conservative, hereafter we consider only the group region within one $R_{\mathrm{V}}$, which contains enough galaxies to work with a large statistical data-base: $\sim 70-80 \%$ of the whole sample, i.e. 1584 and 1882 galaxies for the HG and PG catalogs, respectively. This radius corresponds to $\sim 2-3 \times R_{\text {vir }}$ for $\mathrm{PG}$ and $\mathrm{HG}$ respectively, and thus is not too much larger than the virialized region, i.e. where secondary relaxation-processes of some importance might operate.

\subsection{Segregation effects}

We consider the resulting relations for the two ensemble groups, HG and PG: the groupcentric-distance-magnitude relation $(R-M)$; the groupcentric-distance-morphological type

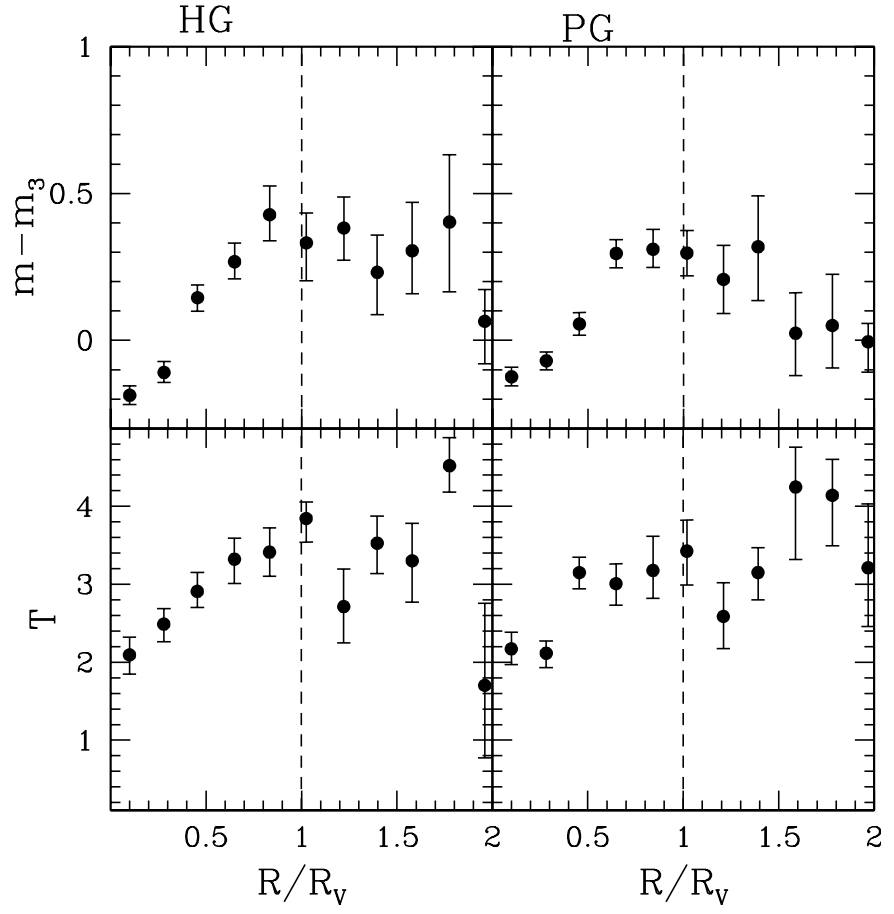

Fig. 2. Results for ensemble groups: (normalized) magnitude $m-m_{3}$, and morphological-type $T$ vs. (normalized) projected group-centric distance $R / R_{\mathrm{V}}$, respectively from top to bottom. Points are biweight mean values with $68 \%$ bootstrap error-bars. The dashed vertical lines indicate $1 \times R_{\mathrm{V}}$, the external limit for following analyses, which corresponds to $\sim 2-3 \times R_{\text {vir }}$ and contains $\sim 70-80 \%$ of all galaxies. This plot is shown to provide a first look at the original data: note that relations involving magnitudes should be properly corrected (cf. Figs. 3 and 4 and Sect. 4.2).

relation $(R-T)$; the velocity-magnitude relation $(V-M)$; and the velocity-morphological type relation $(V-T)$. We analyze segregation effects in the framework of the Kendall rank correlation analysis, which is completely nonparametric (Kendall 1948; cf. also Siegel 1956).

For each interesting relation $x$ vs. $y$, we compute the Kendall coefficient, $K_{x y}$, and the respective significance of the correlation, $P_{x y}$.

When analyzing a correlation, there is always the possibility that this correlation is due to the association between each of the two physical properties and a third property connected to the selection effects of the catalog. Statistically, this problem may be attacked by methods of partial correlation. In partial correlation, the effects of variation by a third $z$ variable upon the relation between the $x$ and $y$ variables are eliminated. In practice, the correlation between the two interesting properties is found with the third variable kept constant. Thus we also compute the Kendall partial correlation coefficient, $K_{x y, z}$, which is a measure of the correlation between two data sets, $x$ and $y$, independently of their correlation with a third data set, $z$ (Kendall 1948; cf. Eq. (9.13) of Siegel 1956):

$K_{x y, z}=\frac{K_{x y}-K_{z y} K_{x z}}{\sqrt{\left(1-K_{z y}^{2}\right)\left(1-K_{x z}^{2}\right)}}$, 
Table 3. Results for ensemble groups.

\begin{tabular}{cccccc}
\hline \hline Sample & $x$ vs. $y$ & $N_{\text {GALs }}$ & \multicolumn{1}{c}{$K_{x y}, P_{x y}$} & \multicolumn{1}{c}{$K_{x y, \bar{v}}, P_{x y, \bar{v}}$} & $K_{x y, n}, P_{x y, n}$ \\
\hline HG Groups & $R-M$ & 1584 & $0.19,>99.9$ & $0.19,>99.9$ & $0.13,>99.9$ \\
& $R-T$ & 1558 & $0.07,>99.9$ & $0.07,>99.9$ & $0.09,>99.9$ \\
& $V-M$ & 1584 & $0.06,>99.9$ & $0.05,>99.9$ & $0.04, \quad 99.6$ \\
& $V-T$ & 1558 & $0.02,84.7$ & $0.02,93.0$ & $0.02,89.3$ \\
PG Groups & $R-M$ & 1882 & $0.16,>99.9$ & $0.16,>99.9$ & $0.09,>99.9$ \\
& $R-T$ & 1856 & $0.06,>99.9$ & $0.06,>99.9$ & $0.06,>99.9$ \\
& $V-M$ & 1882 & $0.06,>99.9$ & $0.06,>99.9$ & $0.03, \quad 99.3$ \\
& $V-T$ & 1856 & $-0.01,67.1$ & $-0.01, \quad 69.0$ & $0.00, \quad 54.4$ \\
\hline
\end{tabular}

where, as for the third variable, we consider both the group distance $(\propto \bar{v})$, and the number of group-members (richness $n)$. In fact, the distance is connected with the selection function of the group catalog, which is based on an apparent magnitude complete galaxy sample. Moreover, in such very poor systems $(n \gtrsim 3)$ the normalized magnitude $m-m_{3}$ strongly correlates with $n$, which in its turn is slightly correlated with both size and velocity dispersion (cf. Table 1).

For both the HG and PG catalogs, Table 3 shows the results for the ensemble groups constructed as described in Sect. 4.1. For each interesting relation $x$ vs. $y$, we give the Kendall coefficient, $K_{x y}$, and the Kendall partial correlation coefficient, which takes into account the effect of the third variable, $K_{x y, \bar{v}}$ and $K_{x y, n}$. We also list the respective significance of the correlations, $P$. The value of $P_{x y}$ is recovered form the fact that the sampling distribution of $K_{x y}$ is practically indistinguishable from the normal distribution (Kendall 1948; cf. Eq. (9.11) of Siegel 1956). Since the sampling distribution of $K_{x y, z}$ is unknown, to compute $P_{x y, \bar{v}}$ and $P_{x y, n}$ we adopt the bootstrap method performing 1000 bootstrap resamplings for each correlation.

The Kendall coefficients of the $R-T$ and $V-T$ correlations show no decrease when the $\bar{v}$ or $n$ variables are taken into account $\left(K_{x y, \bar{v}} \sim K_{x y, n} \sim K_{x y}\right)$. Therefore the $R-T$ and $V-T$ relations are not biased by systematic effects.

As for the $R-M$ and $V-M$ relations, we find that $K_{x y, \bar{v}} \sim K_{x y}$, while $K_{x y, n}$ is systematically smaller than $K_{x y}$ although still giving significant correlation. Thus, we conclude that the $R-M$ and $V-M$ correlations are due to a combination of a true physical effect and a spurious one. The spurious one is due to our stacking of groups with different richness.

To support the above analysis, we also make Monte Carlo simulations performing a random shuffle of galaxy velocities, magnitudes, and morphological types within each individual group. This procedure, which leaves unchanged global group properties and modifies the group internal structure, should destroy any physical segregation effects and reveal the presence of spurious correlations. We construct the "simulated" ensemblegroup by combining together the galaxies of groups resulting from Monte Carlo simulations. In particular, we perform 15 Monte Carlo simulations for each group in order to consider a large (>20000) number of galaxies and thus stabilize the resulting Kendall coefficient, $K_{x y}^{\text {sim }}$. No significant correlation is found for $R-T$ and $V-T$ relations. When considering $R-M$ and $V-M$, the simulated group shows a significant correlation, characterized by a $K_{x y}^{\mathrm{sim}}$ smaller than the coefficient of the original
Table 4. Poor $(n<5)$ groups.

\begin{tabular}{|c|c|c|c|}
\hline Sample & $x$ vs. $y$ & $N_{\text {GALs }}$ & $K_{x y, n}, P_{x y, n}$ \\
\hline \multirow[t]{4}{*}{$\mathrm{HG}$} & $R-M$ & 577 & $0.10,>99.9$ \\
\hline & $R-T$ & 565 & $0.07, \quad 99.4$ \\
\hline & $V-M$ & 577 & 0.00 \\
\hline & $V-T$ & 565 & -0.02 \\
\hline \multirow[t]{4}{*}{ PG } & $R-M$ & 705 & 0.06, \\
\hline & $R-T$ & 693 & 0.04 \\
\hline & $V-M$ & 705 & 0.02 \\
\hline & $V-T$ & 693 & $0.01, \quad 62.5$ \\
\hline
\end{tabular}

data $K_{x y}$. Moreover, when using only groups with a fixed number of members $n=3,4,5$, and 6 (groups with $n>6$ are statically poorly represented) the corresponding Monte Carlo simulated groups show no correlation at all. Thus, in agreement with Kendall partial correlation analysis, we conclude that $R-M$ and $V-M$ correlations are partially spurious due to our stacking of groups with different richness.

Hereafter, to take into accounts the richness effect, we consider as really physical meaningful the $K_{x y, n}$ coefficient.

Having addressed the problem of possible biases, we can give our results about segregation effects, which are similar for both the HG and PG catalogs. Spatial correlations, in luminosity or morphology $(R-M, R-T)$, are the strongest one $\left(K_{x y, n}=0.06-0.13\right)$. Luminosity segregation in velocity $(V-M)$ is characterized by a small correlation coefficient $\left(K_{x y, n}=\right.$ $0.03-0.04)$, still significant. Morphological segregation in velocity $(V-T)$ is not significant, in agreement with the fact that this is the faintest segregation detected in Sect. 3.

The interesting relations are better visualized in Figs. 3 and 4 for both all and rich $n \geq 5$ groups. There, to take into account the spurious component in the $R-M$ and $V-M$ relations, we show our results rescaled to those of the corresponding simulated group, i.e. we normalize the ( $y$-axis) values obtained for the real ensemble-group to the values obtained for the corresponding simulated-group with the same binning procedure. For the sake of homogeneity, we apply the same procedure to $R-T$ and $V-T$ relations.

\subsection{Segregation and group richness}

We analyze separately groups with $n<5$ and those with $n \geq$ 5 members. Tables 4 and 5 show the results of the correlation analysis. 
Table 5. Rich ( $n \geq 5)$ groups.

\begin{tabular}{ccrccl}
\hline \hline Sample & $x$ vs. $y$ & $N_{\mathrm{GALs}}$ & $K_{x y, n}, P_{x y, n}$ & $K_{x y, n, m-m 3}, P_{x y, n, m-m_{3}}$ & $K_{x y, n, T}, P_{x y, n, T}$ \\
\hline HG & $R-M$ & 1007 & $0.13,>99.9$ & & $0.12,>99.9$ \\
& $R-T$ & 993 & $0.10,>99.9$ & $0.09,>99.9$ & \\
& $V-M$ & 1007 & $0.06,99.9$ & & $0.05,99.7$ \\
& $V-T$ & 993 & $0.05,99.4$ & $0.05,98.6$ & \\
PG & $R-M$ & 1177 & $0.09,>99.9$ & & $0.08,>99.9$ \\
& $R-T$ & 1163 & $0.10,>99.9$ & $0.09,>99.9$ & \\
& $V-M$ & 1177 & $0.03,92.4$ & & $0.03,93.1$ \\
& $V-T$ & 1163 & $0.00,58.5$ & $-0.01,64.7$ & \\
\hline
\end{tabular}

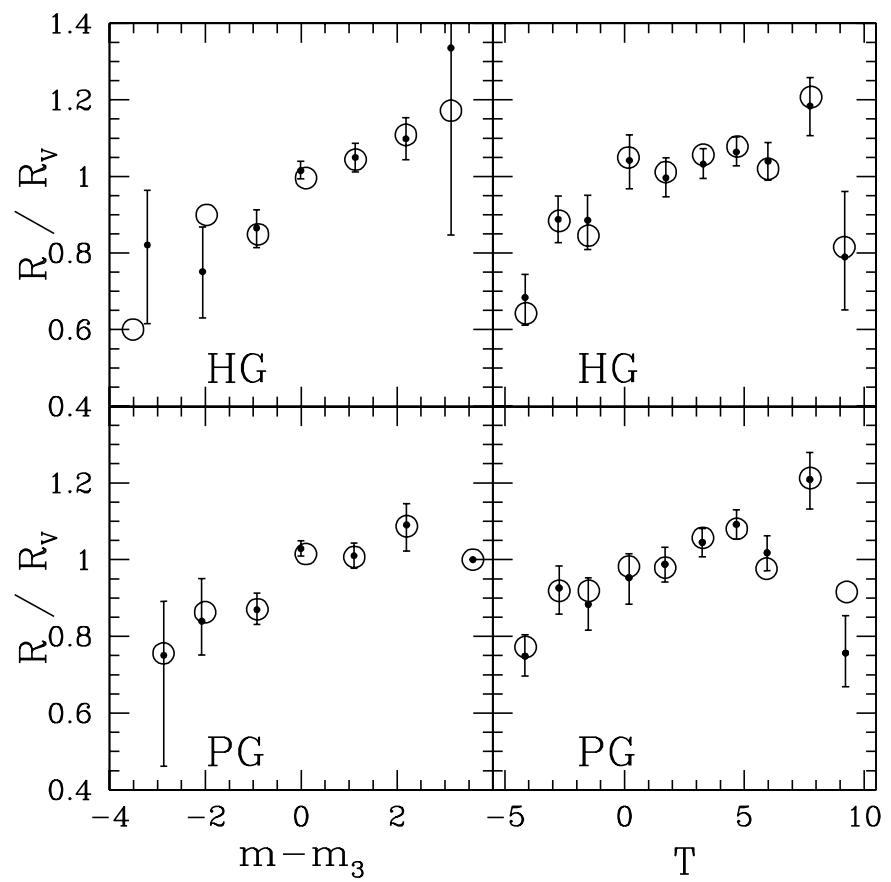

Fig. 3. Groupcentric-distance vs. magnitude, and morphological type: $R-M$ and $R-T$ relations in the left and right panels, respectively. Points are biweight mean values for all groups (filled circles) and rich $n \geq 5$ groups only (open circles). Error bars are $68 \%$ bootstrap estimates. For the sake of clarity, error bars are shown for one sample only. Observational results are normalized point by point with results from simulated groups (cf. text).

The analysis of rich groups confirms the correlations found for the whole sample. In particular, the $V-T$ correlation is now significant in the case of the $\mathrm{HG}$ catalog.

As for poor groups, they show fainter spatial segregation effects than rich groups and no kinematical segregation effects at all (cf. also Fig. 5). This result could be due 1) to the dilution effect of a significant number of spurious groups or 2) to some physical difference between poor and rich groups. The most relevant difference is probably the $\sigma_{\mathrm{v}}$, which is, on average, smaller in poor than in rich groups (cf. Table 1). However, segregation effects are poorly or not dependent on $\sigma_{\mathrm{v}}$ (cf. Sect. 4.5); thus we are inclined to believe in the first hypothesis.

Hereafter, we consider only more reliable, rich $n \geq$ 5 groups.

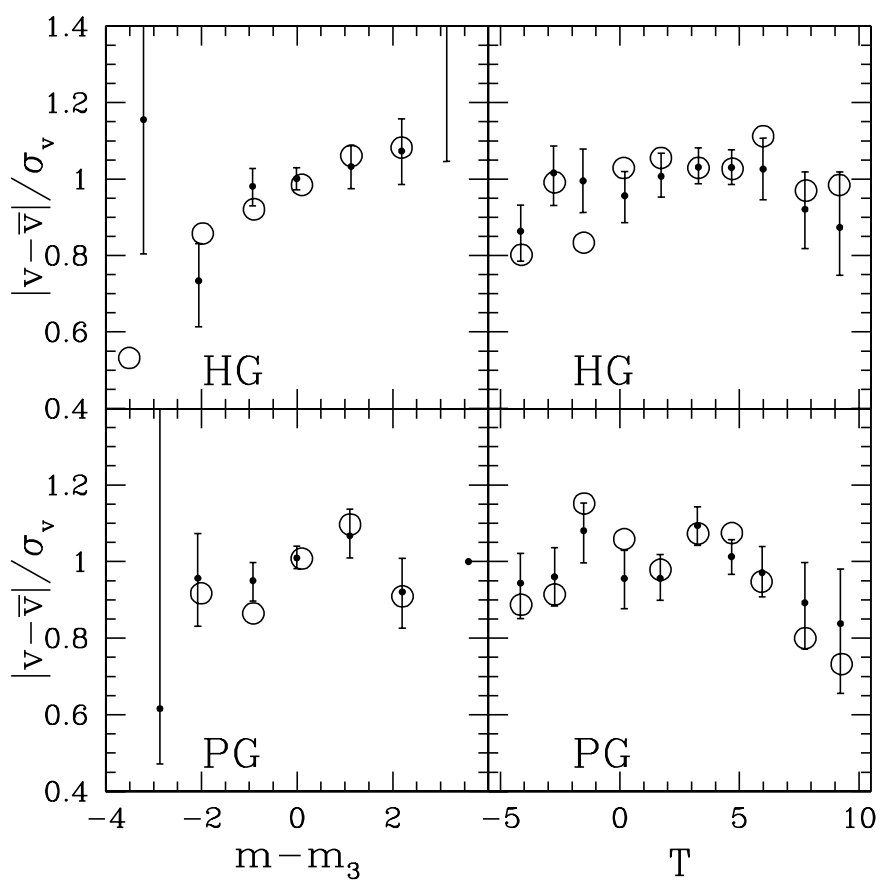

Fig. 4. Velocity vs. magnitude, and morphological type: $V-M$ and $V-T$ relations in the left and right panels, respectively. Points are biweight mean values for all groups (filled circles) and rich $n \geq$ 5 groups only (open circles). Error bars are $68 \%$ bootstrap estimates. For the sake of clarity, error bars are shown for one sample only. Observational results are normalized point by point with results from simulated groups (cf. text).

\subsection{Luminosity vs. morphology effects}

It is well known that galaxies of different morphological types have different luminosity functions (e.g., Sandage et al. 1985; Marzke et al. 1998). In particular, very late-type galaxies strongly differ from other types having typically fainter magnitudes (e.g., Sandage et al. 1985; Sandage 2000). In our case, morphological type $T$ and normalized magnitude $m-m_{3}$ correlate at the $98.8 \%$ and $97.8 \%$ c.l. for $\mathrm{HG}$ and PG, respectively.

The problem of the independence of morphology and luminosity segregations can be addressed by methods of partial correlation (cf. Sect. 4.2). Thus, for the four relations considered in Table 5 we estimate the Kendall partial rank correlation coefficient considering the effect of luminosity (morphology) in the relations involving morphology (luminosity). Table 5 lists the values of $K_{x y, n, m-m_{3}}$ and $K_{x y, n, T}$, and the respective c.l.: they are similar to or just slightly smaller than the values of 


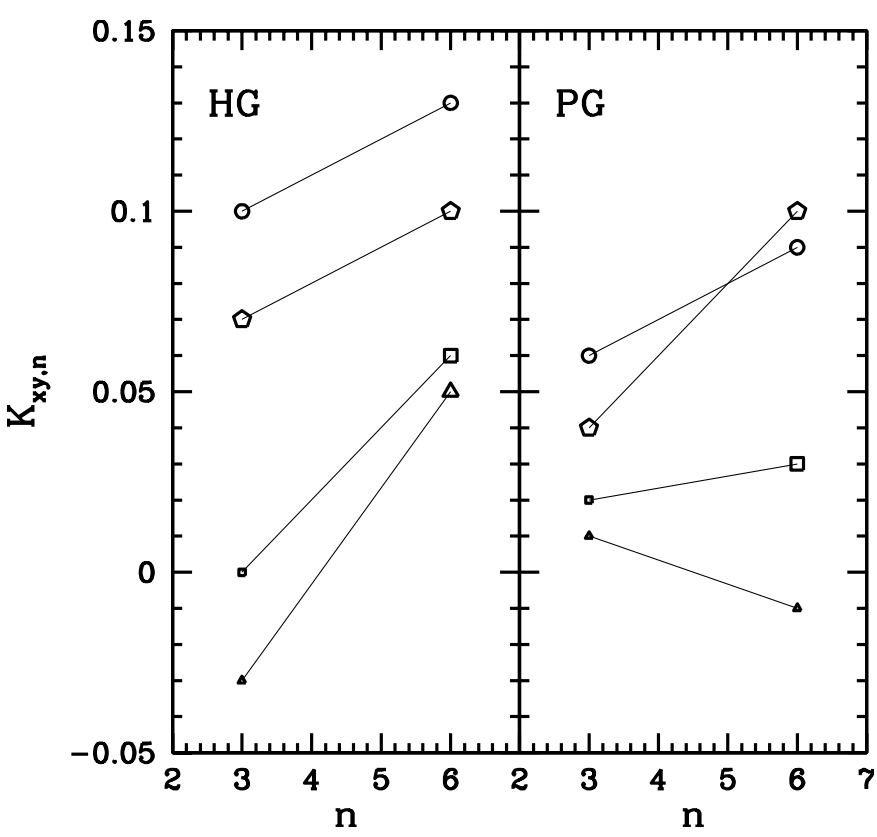

Fig. 5. Values of Kendall-correlation coefficients $K_{x y, n}$ for poor $(n<5)$ and rich $(n \geq 5)$ groups $(n=3$ and 6 are the median values for poor and rich groups, respectively). The results for all four relations are shown: $R-M$ (circles), $R-T$ (pentagons), $V-M$ (squares), and $V-T$ (triangles). Larger symbols indicate a correlation with significance $\geq 90 \%$.

Table 6. Luminosity vs. morphology segregation.

\begin{tabular}{|c|c|c|c|c|}
\hline$x$ vs. $y$ & $N_{\mathrm{GALs}}$ & $K_{x y, n}, P_{x y, n}$ & $N_{\mathrm{GALs}}$ & $K_{x y, n}, P_{x y, n}$ \\
\hline & \multicolumn{2}{|c|}{ HG early-types } & \multicolumn{2}{|c|}{ HG late-types } \\
\hline$R-M$ & 267 & $0.10, \quad 99.7$ & 726 & $0.13,>99.9$ \\
\hline \multirow[t]{2}{*}{$V-M$} & 267 & $0.07, \quad 96.9$ & 726 & $0.05, \quad 98.4$ \\
\hline & \multicolumn{2}{|c|}{ PG early-types } & \multicolumn{2}{|c|}{ PG late-types } \\
\hline$R-M$ & 344 & $0.08, \quad 98.6$ & 819 & $0.08,>99.9$ \\
\hline$V-M$ & 344 & $0.10, \quad 99.9$ & 819 & $-0.01, \quad 63.5$ \\
\hline
\end{tabular}

corresponding $K_{x y, n}$. We conclude that morphology and luminosity segregations are two independent effects.

Moreover, we consider early- and late-type galaxies separately (cf. Table 6). The $R-M$ correlation is significant for both early- and late-type samples. The same is true for the $V-M$ correlation in the HG case, but not in the PG case, where only the early-type sample shows a significant $V-M$ correlation.

\subsection{Segregation and velocity dispersion}

The price to be paid for the large gain in statistics when using the above ensemble groups is that we average away possible distinctive behaviors. In particular, in the NOG sample, the value of velocity dispersion ranges from very low- $\sigma_{\mathrm{v}}$ systems up to systems with $\sigma_{\mathrm{v}} \sim 350-500 \mathrm{~km} \mathrm{~s}^{-1}$ (cf. Fig. 1).

We consider the four quartiles of the distribution of $\sigma_{\mathrm{v}}$-values, i.e. each with $\sim 37$ and $\sim 42$ groups for HG5 and PG5, respectively. The related ensemble systems contain 184-341 galaxies for HG5 and 223-371 galaxies for PG5. Figure 6 shows the resulting Kendall coefficient for these ensemble systems. No systematic, general evidence of a

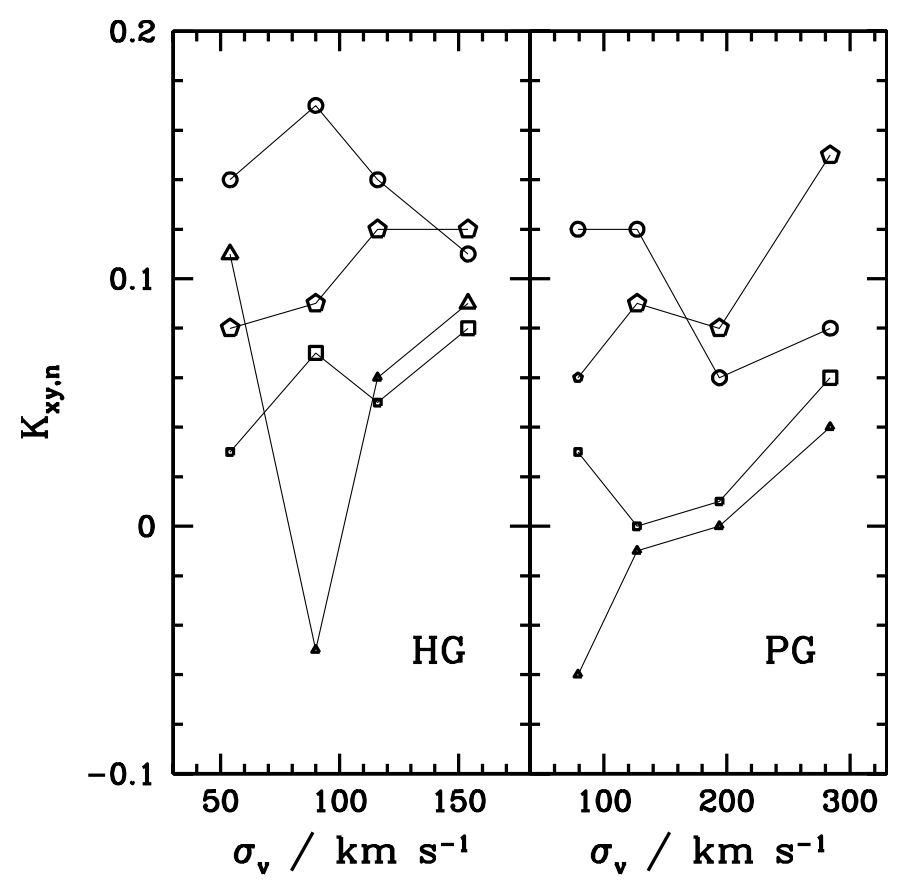

Fig. 6. Values of Kendall-correlation coefficients $K_{x y, n}$ as a function of $\sigma_{\mathrm{v}}$ (here the median values in each quartile, cf. Fig. 1). The results for all four relations are shown: $R-M$ (circles), $R-T$ (pentagons), $V-M$ (squares), and $V-T$ (triangles). Larger symbols indicate a correlation with significance $\geq 90 \%$.

connection between segregation properties and velocity dispersion is shown.

\section{Summary and discussion}

We analyze $\sim 2 \times 400$ loose groups identified in the NOG catalog by using hierarchical and "friends-of-friends" percolation catalogs by $\mathrm{G} 00$.

Analyzing both catalogs we find similar results: earliertype (brighter) galaxies are more clustered and lie closer to the group centers, both in position and in velocity, than later-type (fainter) galaxies. Spatial segregations are stronger than kinematical segregations. These effects are generally detected at the $\gtrsim 3$-sigma level, with the exception of morphological segregation in velocity (cf. Sects. 3 and 4.2). The significance of the last effect is generally lower than $99 \%$ in the comparison between weighted and unweighted properties (cf. Sect. 3), and it is confirmed only for the HG rich ensemble-group (cf. Sect. 4.3).

Our main results are confirmed by the analysis of statistically more reliable $\sim 2 \times 150$ rich groups (with at least five members, cf. Sect. 4.3). Results for poorer groups are possibly diluted by spurious systems.

The evidence of spatial morphology-segregation confirms previous results recovered by using directly morphology data or morphological indicators such as spectral types and colors (e.g., Postman \& Geller 1984; Mahdavi et al. 1999; Tran et al. 2001; Carlberg et al. 2001b; Domínguez et al. 2002). The other kinds of segregation we detect are still poorly analyzed or debated questions for groups, but are studied in the context of galaxy clusters. 


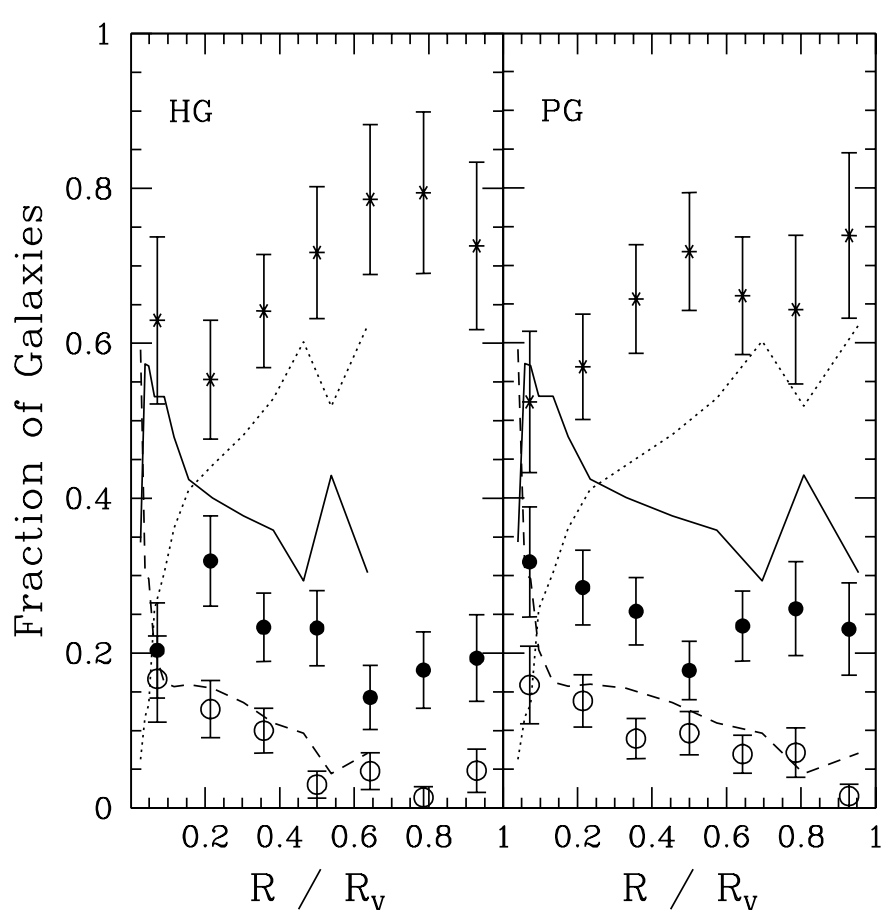

Fig. 7. Fraction of different morphological-types as a function of distance from the group center: ellipticals (open circles), lenticulars (filled circles), and spirals + irregulars (stars). Points are biweight mean values with $68 \%$ Poissonian error-bars. Results for rich $n \geq$ 5 groups are shown. Whitmore et al. 1993 data for ellipticals (dashed line), lenticulars (solid line), and spirals + irregulars (dotted line) are also shown for a qualitative comparison only (see text).

Recent observational efforts to increase the number of members of a few loose groups suggest that they are quasivirialized system, at least in their central regions, and show a continuum of properties with respect to clusters, e.g., regarding the density profile of galaxy distribution, the behavior of velocity dispersion profiles, and the presence of substructures (Mahdavi et al. 1999; Zabludoff \& Mulchaey 1998a,b). In this context, it is worth to attempt a more detailed comparison between our results for groups and those obtained for clusters.

In Fig. 7 we plot the relative fraction of ellipticals $(T<$ $-3.5)$, lenticulars $(-3.5 \leq T<0)$, and spirals + irregulars $(T \geq 0)$ for ensemble systems constructed from rich groups $(n \geq 5)$. The qualitative behavior resembles that already found for clusters (e.g. Whitmore et al. 1993): the fractions of ellipticals and lenticulars decline with radius and the fraction of late-type galaxies increases. For the sake of completeness we also plot cluster data by Whitmore et al. which correspond to $\sim 2 R_{\text {vir }}$ (i.e. $\sim 1$ and $2 / 3 R_{\mathrm{V}}$ for PG and $\mathrm{HG}$, respectively, cf. Sect. 4.1). Due to the different depth of the catalogs, the possible inconsistencies in the morphological classification criteria, and the degree of uncertainty in the normalization radius we do not attempt a more quantitative comparison.

In agreement with cluster studies (e.g., Biviano et al. 2002), we find significant evidence of both spatial and kinematical segregation of galaxies of different luminosity, and luminosity segregation seems to be related only to the most luminous galaxies. Figure 8 directly compares the $V-M$ relation for NOG groups to that for clusters by Biviano et al. (1992) who

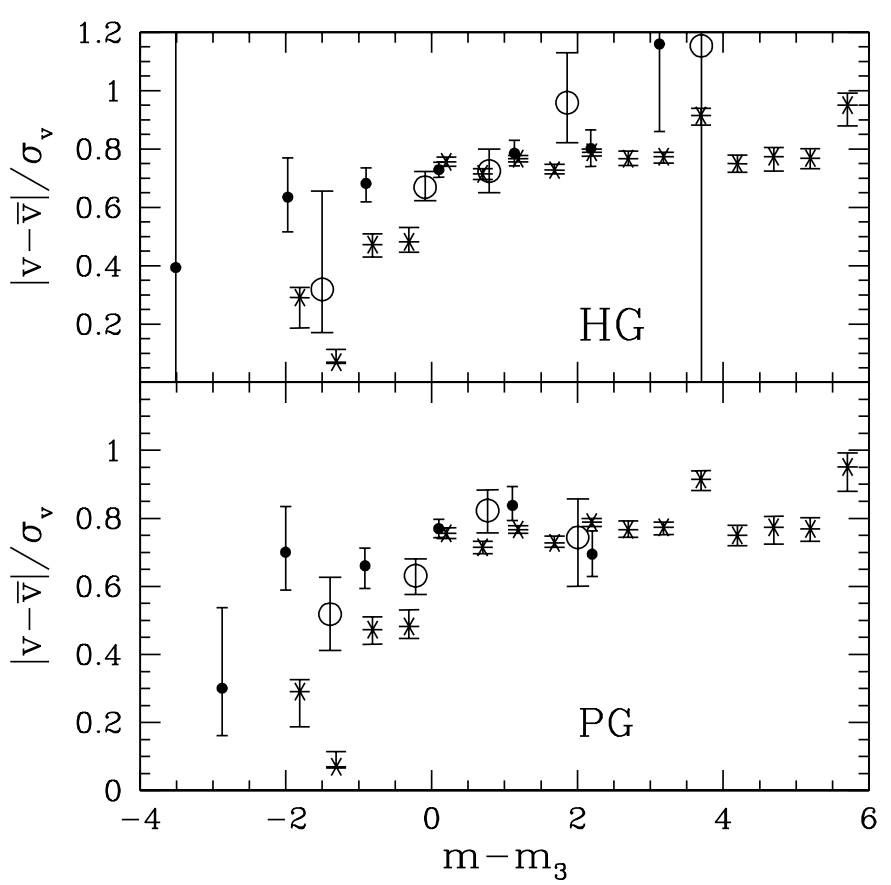

Fig. 8. $V-M$ relation of rich groups for all galaxies (filled circles) and only for early-type galaxies (open circles). It is compared to the $V-M$ relation of clusters (stars, Biviano et al. 1992). Points are biweight mean values with $68 \%$ bootstrap error-bars. Observational results for groups are normalized point by point with results from simulated groups as in Fig. 3 and then rescaled to the average value obtained from simulations.

found that the $V-M$ relation first rapidly increases and then flattens out at faint magnitudes. According to Biviano et al., this trend can be explained if galaxies brighter than $m_{3}$ have achieved the energy-equipartition status, maybe due to dynamical friction or galaxy merging, while fainter galaxies still lie in the velocity-equipartition status generated by violent relaxation. NOG groups show a less sharp increase in the region of bright galaxies with respect to clusters. A somewhat better agreement is obtained when considering only early-type galaxies, which might better represent the typical morphological content of those clusters. Another explanation is that the removing of late-type galaxies reduces the number of possible non-member galaxies highlighting better the true physical relation. Deeper samples for groups would be needed to verify the flatness of the relation at faint magnitudes.

In NOG groups we find that luminosity segregation is found independent of morphological segregation in agreement with the results from clusters (e.g., Adami et al. 1998; Biviano et al. 2002). In clusters luminosity segregation is evident only for ellipticals and possibly lenticulars (e.g., Stein 1997; Biviano et al. 2002). In groups we do not reach a definitive conclusion since we find that spatial luminosity segregation concerns both early- and late-type galaxies, while kinematical luminosity segregation seems confined to early-type galaxies (in the case of PG groups, cf. Sect. 4.4).

Recent results suggest that morphological segregation in space characterizes only massive groups, above some threshold value. In fact, Carlberg et al. (2001b) found the presence 
of a color gradient only in groups with $\sigma_{\mathrm{v}}>150 \mathrm{~km} \mathrm{~s}^{-1}$, and Domínguez et al. (2002) detected spectral-type segregation only in groups with mass $\mathcal{M} \gtrsim 10^{13.5} h^{-1} \mathcal{M}_{\odot}$. Additional support to this idea is given by our analysis in Sect. 4.5 , where the $R-T$ relation is strengthened with increasing velocity dispersion (cf. pentagons in Fig. 6). However, no systematic, general evidence of a connection between segregation properties and velocity dispersion is shown and the question merits further investigations.

To sum up, as regards coarse aspects of morphology and luminosity segregation, our results are consistent with a continuum of properties of galaxies in systems, from low-mass groups to massive clusters. This result is in agreement with the early study by Postman \& Geller (1984) on morphological segregation and, e.g., more recent results by Lewis et al. (2001) who found that environmental influences on galaxy star formation are not restricted to cluster cores, but are effective in groups, too.

Our results suggest that the segregation effects we analyze are mainly connected with the initial conditions at the time of galaxy formation. Alternatively, the mechanisms which influence galaxy luminosity and morphology should act in a similar way in groups and in clusters, or, at least, in the subunits forming clusters in the context of the hierarchical scenario.

In this framework, it is also worth discussing our results in connection to those coming from large-scale analyses, since a large fraction of galaxies is located in groups $(\sim 40 \%$, cf. Ramella et al. 2002). Spatial segregation phenomena are generally studied through the galaxy-galaxy correlation function analysis. This kind of analysis is fully independent and complementary to our work, which concerns very small scales. As for NOG catalog, Giuricin et al. (2001) analyzed the redshiftspace two-point correlation function of galaxies, finding that - on scales between $\sim 3$ and 10-20 $h^{-1} \mathrm{Mpc}$ - the strength of clustering is stronger for earlier and, independently, for more luminous galaxies, in agreement with most recent literature (cf. Giuricin et al. and refs. therein). The projected correlation function overcomes the problem of distortions of the clustering pattern induced by peculiar motions and allows to perform analysis down to smaller scales $\left(\lesssim 0.5 h^{-1} \mathrm{Mpc}\right)$. In particular, very recent studies of the $2 \mathrm{dF}$ Galaxy Redshift Survey show that the strength of the clustering of luminous galaxies increases with the galaxy luminosity and that luminosity and spectraltype segregations are independent effects (Norberg et al. 2001, 2002). Thus, segregation phenomena, qualitatively in agreement with those detected in our study, are present out to very large scales. This could be another piece of evidence in favor of a mostly primordial origin for spatial segregation effects.

Acknowledgements. We thank Andrea Biviano, Manuela Magliocchetti, Christian Marinoni, and Massimo Ramella for useful suggestions and discussions. We also thank the anonymous referee for a careful reading of the manuscript and suggestions which improved the paper. Work partially supported by the Italian Ministry of Education, University, and Research (MIUR, grant COFIN2001028932 "Clusters and groups of galaxies, the interplay of dark and baryonic matter"), and by the Italian Space Agency (ASI).

\section{References}

Adami, C., Biviano, A., \& Mazure, A. 1998, A\&A, 331, 439

Antonuccio-Delogu, V., Becciani, U., van Kampen, E., et al. 2002, MNRAS, 332, 7

Bahcall, N. A., Lubin, L. M., \& Dorman, V. 1995, ApJ, 447, L81

Beers, T. C., Flynn, K., \& Gebhardt, K. 1990, AJ, 100, 32

Benoist C., Maurogordato S., da Costa L. N., Cappi A., \& Schaffer R., 1996, ApJ, 472, 452

Bhavsar, S. P. 1981, ApJ, 246, L5

Binggeli, B., Popescu, C. C., \& Tammann, G. A. 1993, A\&AS, 98, 275

Binggeli, B., Tammann, G. A., \& Sandage, A. 1987, AJ, 94, 251

Binney, J., \& Tremaine, S. 1987, Galactic Dynamics (Princeton Univ. Press)

Biviano, A., Katgert, P., Mazure, A., et al. 1997, A\&A, 321, 84

Biviano, A., Katgert, P., Thomas, T., \& Adami, C. 2002, A\&A, 387, 8

Biviano, A., Girardi, M., Giuricin, G., Mardirossian, F., \& Mezzetti, M. 1992, ApJ, 396, 35

Carlberg, R. G., Yee, H. K. C., Ellingson, E., et al. 1997, ApJ, 478, 462

Carlberg, R. G., Yee, H. K. C., Morris, S. L., et al. 2001a, ApJ, 552, 427

Carlberg, R. G., Yee, H. K. C., Morris, S. L., et al. 2001b, ApJ, 563, 736

Danese, L., de Zotti, G., \& di Tullio, G. 1980, A\&A, 82, 322

de Souza, R. E., Capelato, H. V., Arakaki, L., \& Logullo, C. 1982, ApJ, 263, 557

de Theije, P. A. M. \& Katgert, P. 1999, A\&A, 341, 371

de Vaucouleurs, G., de Vaucouleurs, A., Corwin, et al. 1991, Third Reference Catalogue of Bright Galaxies (Berlin Heidelberg New York: Springer-Verlag) (RC3)

Diaferio, A., Kauffmann, G., Colberg, J. M., \& White, S. D. M. 1999, MNRAS, 307, 537

Domínguez, M., Zandivarez, A. A., Martínez, H. J., et al. 2002, MNRAS, 335, 825

Dressler, A. 1980, ApJ, 236, 351

Dressler, A., Oemler, A. Jr., Couch, W. J., et al. 1997, ApJ, 490, 577

Frederic, J. J. 1995, ApJS, 97, 275

Garcia, A. M. 1993, A\&AS, 100, 47

Girardi, M., \& Giuricin, G. 2000, ApJ, 540, 45

Girardi, M., Giuricin, G., Mardirossian, F., Mezzetti, M., \& Boschin, W. 1998, ApJ, 505, 74

Giuricin, G. P., Mardirossian, F., \& Mezzetti, M. 1982, ApJ, 255, 361

Giuricin, G., Mardirossian, F., Mezzetti, M., Pisani, A., \& Ramella, M. 1988, A\&A, 192, 95

Giuricin, G., Marinoni, C., Ceriani, L., \& Pisani, A. 2000, ApJ, 543, 178 (G00)

Giuricin, G., Samurović, S., Girardi, M., Mezzetti, M., \& Marinoni, C. 2001, ApJ, 554, 857

Gourgoulhon, E. , Chamaraux, P., \& Fouqué, P. 1992, A\&A, 255, 69

Gunn, J. E., \& Gott, J. R. I. 1972, ApJ, 176, 1

Guzzo, L., Strauss, M. A., Fisher, K. B., Giovanelli, R., \& Haynes, M. P. 1997, ApJ, 489, 37

Hermit S., Santiago B. X., Lahav O., et al., 1996, MNRAS, 283, 709

Huchra, J. P., \& Geller, M. J. 1982, ApJ, 257, 423

Kendall, M. 1948, Rank Correlation Methods (London: Griffin)

Kendall, M, \& Stuart, A. 1979, in Advanced Theory of Statistics, ed. Buther \& Tonner Ltd, 546

Ledermann, W. 1982, Handbook of Applicable Mathematics (New York: Wiley), Vol. 6

Lewis, I., Balogh, M., De Propris, R., et al. 2002, MNRAS, 334, 673

Lynden-Bell, D. 1967, MNRAS, 136, 101

Magtesyan, A. P. \& Movsesyan, V. G. 1995, Astron. Lett., 21, 429 
Mahdavi, A., Böhringer, H., Geller, M. J., \& Ramella, M. 1997, ApJ, 483,68

Mahdavi, A., Geller, M. J., Böhringer, H., Kurtz, M. J., \& Ramella, M. 1999, ApJ, 518, 69

Makino, J., \& Hut, P. 1997, ApJ, 481, 83

Maia, M. A. G., \& da Costa, L. N. 1990, ApJ, 352, 457

Marzke, R. O., da Costa, L. N., Pellegrini, P. S., Willmer, C. N. A., \& Geller, M. J. 1998, ApJ, 503, 617

Mezzetti, M., Pisani, A., Giuricin, G., \& Mardirossian, F. 1985, A\&A, 143,188

Moore, B., Katz, N., Lake, G., Dressler, A., \& Oemler, A. 1996, Nature, 379, 613

Moss, C. 2001, in Galaxy Disks and Disk Galaxies, ed. J. G. Funes, S. J. \& E. M. Corsini (San Francisco: Astronomical Society of the Pacific), ASP Conf. Ser., 230, 487

Moss, C. \& Dickens, R. J. 1977, MNRAS, 178, 701

Nolthenius, R., Klypin, A., \& Primack, J. R. 1997, ApJ, 480, 43

Norberg, P., Baugh, C. M., \& Hawkins, E. et al. 2001. MNRAS, 328, 64

Norberg, P., Baugh, C. M., \& Hawkins, E. et al. 2002. MNRAS, 332, 827

Oemler, A. J. 1974, ApJ, 194, 1

Ozernoy, L. M., \& Reinhardt, M. 1976, A\&A, 52, 31

Paturel, G., Andernach, H., Bottinelli, L., et al. 1997, A\&AS, 124, 109

Pisani, A., Giuricin, G., Mardirossian, F., \& Mezzetti, M. 1992, ApJ, 389,68

Postman, M., \& Geller, M. J. 1984, ApJ, 281, 95
Ramella, M., Geller, M. J., \& Huchra, J. P. 1989, ApJ, 344, 57

Ramella, M., Geller, M. J., Huchra, J. P., \& Thorstensen, J. R. 1995, AJ, 109, 1469

Ramella, M., Geller, M. J., Pisani, A., \& da Costa, L. N. 2002, AJ, 123, 2976

Ramella, M., Pisani, A., \& Geller, M. J. 1997, AJ, 113, 483

Richstone, D. 1990, in Cluster of Galaxies, ed. W. R. Oegerle, M. J. Fitchett, \& L. Danly (Cambridge: Cambridge University Press), 231

Sandage, A., 2000, PASP112, 504

Sandage, A., Binggeli, B., \& Tammann, G. A. 1985, AJ90, 1759

Sarazin, C. L. 1986, Rev. Modern Phy., 58, 1

Siegel S. 1956, in Nonparameteric statistics for the behavioral sciences, International Student Edition - McGraw-Hill Series in Psychology (Tokyo: McGraw-Hill Kogakusha)

Stein, P. 1997, A\&A, 317, 670

Thompson, W. R. 1936, Ann. Math. Statist., 7, 122

Tran, K. H., Simard, L., Zabludoff, A. I., \& Mulchaey, J. S. 2001, ApJ, 549,172

Tully, R. B. 1987, ApJ, 321, 280

Tucker, D. L., Oemler, A. Jr., Hashimoto, Y., et a. 2000 ApJS, 130, 237

White, S. D. M. 1983, Nature, 302, 756

Whitmore, B. C., Gilmore, D. M., \& Jones, C. 1993, ApJ, 407, 489

Zabludoff, A. I. \& Mulchaey, J. S. 1998a, ApJ, 496, 39

Zabludoff, A. I. \& Mulchaey, J. S. 1998b, ApJ, 498, L5 